Regular Article

\title{
Microswimmers near surfaces
}

\author{
Jens Elgeti ${ }^{\mathrm{a}}$ and Gerhard Gompper ${ }^{\mathrm{b}}$ \\ Theoretical Soft Matter and Biophysics, \\ Institute of Complex Systems \& Institute for Advanced Simulation, \\ Forschungszentrum Jülich, D-52425 Jülich, Germany
}

Received 4 March 2016 / Received in final form 17 May 2016 Published online 10 November 2016

\begin{abstract}
Both, in their natural environment and in a controlled experimental setup, microswimmers regularly interact with surfaces. These surfaces provide a steric boundary, both for the swimming motion and the hydrodynamic flow pattern. These effects typically imply a strong accumulation of microswimmers near surfaces. While some generic features can be derived, details of the swimmer shape and propulsion mechanism matter, which give rise to a broad range of adhesion phenomena and have to be taken into account to predict the surface accumulation for a given swimmer. We show in this minireview how numerical simulations and analytic theory can be used to predict the accumulation statistics for different systems, with an emphasis on swimmer shape, hydrodynamics interactions, and type of noisy dynamics.
\end{abstract}

\section{Introduction}

Lord Rothschild discovered already in 1963, more than 50 years ago, that live human sperm cells accumulate at surfaces, while dead cells do not [1]. Thus, classical attractive interactions, like electrostatic and van der Waals interactions, or interactions mediated by receptor-ligand binding, are unlikely candidates for this surface accumulation. Since then, a similar behaviour has been observed for several other swimming micro-organisms, such as sperm of other species like bull [2] and sea-urchin, and bacteria like $E$. coli $[3,4]$. Thus, surface accumulation seems to be a generic behaviour of microswimmers. This calls for a general physical description of this phenomenon [5]. Furthermore, although surface accumulation seems to be generic, it does not have to be identical for all self-propelled particles. They could be closer to the surface or further away, and their retention time at the surface can be long or short.

A detailed understanding of the surface accumulation of microswimmers is relevant in a large number of current and future applications [5-8]. Biological micro-organisms find many surfaces in their natural environments. For example, mammal sperm has to move through the oviduct to reach the egg. Sperm of many fishes can enter the egg only at a particular spot, the micropyle, and thus has to locate it on the surface. Bacteria move along surfaces where they form colonies and biofilms [9]. Surfaces, in

\footnotetext{
a e-mail: j.elgeti@fz-juelich.de

b e-mail: g.gompper@fz-juelich.de
} 

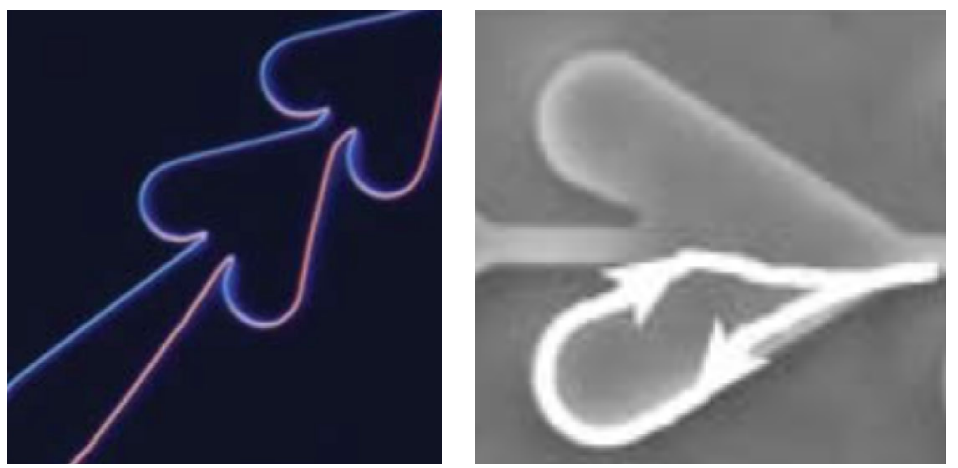

Fig. 1. Clover-leaf structures in microchannels can be employ to rectify microswimmer motion. Particles moving in the wrong direction follow the surface of the leaf at are thereby redirected by $180^{\circ}$. From Ref. [10]. (This figure is subject to copyright protection and is not covered by a Creative Commons license.)

particular in microfluidic devices, can also be used to control and direct the motion of natural and artificial microswimmers [5]. For example, clover-shaped segments in a microchannel can work as a rectifier [10], see Fig. 1. Also, swimming along surfaces strongly affects motion in curved microchannels [11], which can be used for particle sorting.

How do microswimmers interact with surfaces? Several mechanisms are possible. The first and probably most obvious way of a self-propelled particle to encounter a surface is by self-propulsion. As an active particle moves through the embedding medium, or on a substrate, it will sooner or later approach a confining wall, and will collide with it. Now, an active particle typically is subject to some noise, which can be either passive (thermal) or active. Therefore, it has some orientational persistence, i.e. it keeps pushing in the same direction for some time related to the inverse rotational diffusion constant. During this time, the particle remains at the wall or moves parallel to the wall, which implies an increased particle density near or at the wall. Thus, the competition of self-propulsion and orientational fluctuations induces an effective wall adhesion. This behavior strongly depends on the particle shape and type of orientational dynamics, as discussed in detail in Sect. 2. Here, highly idealized model systems are considered, which are employed to elucidate general physical principles of self-propulsion and shape. Self-propelled Brownian rods are discussed in Sect. 2.1, active Brownian spheres in Sect. 2.2, active asymmetric Brownian dumbbells in Sect. 2.3, and run-and-tumble particles in Sect. 2.4.

It is worth mentioning that active Brownian particles have become a standard model of self-propelled particles with noisy dynamics, in particular for studies of motility-induced phase separation and collective dynamics, see recent reviews [5,12], and minireview [13] by T. Speck in this issue.

A second mechanisms is due to hydrodynamic interactions. As a microswimmer usually swims in an aqueous environment, it sets the surrounding fluid into motion as it pushes itself forward. Since the fluid motion is affected by the presence of nearby boundaries, the fluid mediates an interaction between microswimmers and walls. The effects of hydrodynamic interaction on the surface accumulation and swimming behavior are described in Sect. 3. This interaction is rather simple in the far-field, when the distance of the microswimmer from the wall is much larger than the size of the microswimmer itself, but becomes rather complex as the microswimmer comes close to the surface, as discussed in Sect. 3.1. In particular, the behavior now depends on the details of the swimming mechanism, such as the snake-like motion of sperm cells, or the corkscrew-like motion of bacteria flagella, as discussed in Sects 3.2 and 3.3, respectively. 

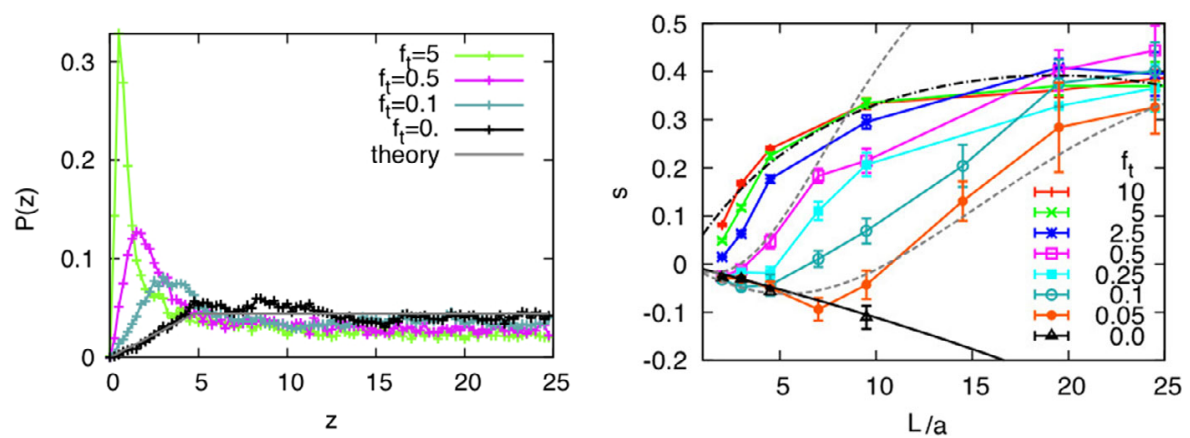

Fig. 2. (Left) Probability density $P(z)$ as function of the distance $z$ from the surface, for various propelling forces $f_{t}$. The rod length is $L \simeq 10 a$ (where $a$ is the rod diameter), the walls are located at $z=0$ and $z=5 L$. A solid gray line shows the density profile of passive rods. (Right) Surface excess $s$ as a function of scaled rod length $L / a$, for various propelling forces $f_{t}$, as indicated. The (black) dashed-dotted line is the scaling result in the ballistic regime (see Eq. (4)), the (gray) dashed lines are scaling results in the diffusive regime for $f_{t}=0.5$ and $f_{t}=0.05$. From Ref. [15]. (This figure is subject to copyright protection and is not covered by a Creative Commons license.)

\section{Active Brownian particles near surfaces: Self-Propulsion, noise, and shape matter}

\subsection{Self-propelled rods near surfaces}

In order to elucidate the effect of shape on the wall adhesion of a self-propelled particle due to steric interactions, it is interesting to consider the behaviour of self-propelled Brownian rods near walls - in the absence of any hydrodynamic interactions, but with Brownian noise (see also minireview [14] by F. Peruani in this issue). In this case, excluded-volume interactions favor parallel orientation along the wall, while the thermal noise leads to fluctuations of the rod orientation and thereby an effective repulsion from the wall. The competition of these two effects gives rise to an interesting adsorption behaviour $[15,16]$.

Very close to the wall, the rod has access to less orientational conformations. This leads to an entropic repulsion from the wall for passive Brownian rods. Results of Brownian Dynamics simulations [15] are shown in Fig. 2. While passive rods are depleted from the surface as expected, active rods show an increased probability density near the surface, which grows with increasing propelling force density $f_{t}$, see Fig. 2(left). In addition to the propelling force, the behaviour of the rods strongly depends on the rod length $L$. The surface excess - the integrated probability density to find a rod near the surface compared to a uniform density distribution - is shown in Fig. 2(right). The results show that (i) propulsion always leads to an increases in surface excess, and (ii) depending on $f_{t}$, the surface excess initially decreases with increasing $L$, but than increases again to reach a plateau nearly independent of $f_{t}$ and $L$ for large propelling forces and rod lengths [15]. In comparison with active Brownian spheres (see Sect. 2.2 below), it is interesting to note that for rods, the surface excess never reaches unity. Thus, the elongated shape leads to a reduction of surface accumulation due to rapid alignment with the surface upon collision.

In order to understand the mechanism which is responsible for the effective surface adhesion of self-propelled rods, and to predict their behaviour as a function of rod length, propulsive force, and wall separation, the analogy of the trajectories of selfpropelled rods with the conformations of semi-flexible polymers can be exploited [15]. 


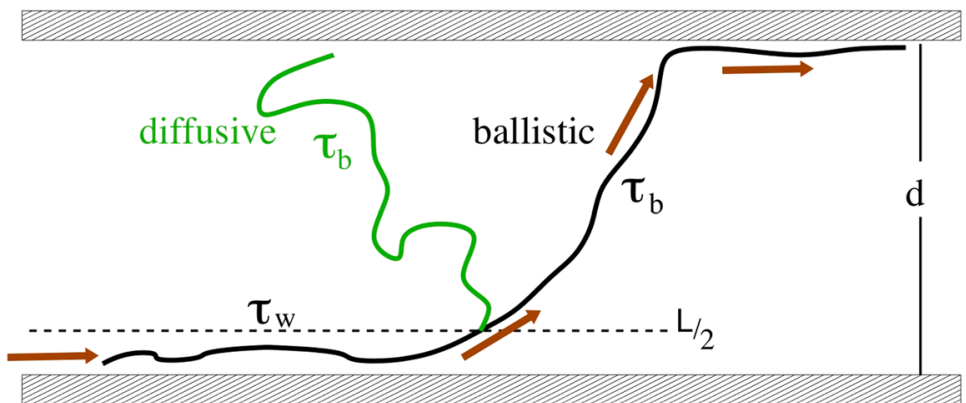

Fig. 3. Schematic representation of the different regimes of rod motion, near a wall for time $\tau_{w}$, and in the bulk for time $\tau_{b}$, either in the ballistic or in the diffusive regime. From Ref. [15]. (This figure is subject to copyright protection and is not covered by a Creative Commons license.)

In the bulk, the rotational diffusion constant of a rod is $D_{R} \sim k_{B} T /\left(\eta L^{3}\right)$, which implies a persistence length

$$
\xi_{p} \sim v / D_{R} \sim \eta v L^{3} / k_{B} T
$$

of the trajectory. The probability $p$ to find the self-propelled rod in a layer of thickness $L / 2$ near the wall can be expressed as $p=\tau_{w} /\left(\tau_{w}+\tau_{b}\right)$, where $\tau_{w}$ is the time the rod remains within this layer and $\tau_{b}$ is the time it is located in the bulk (with $L / 2<z<$ $d-L / 2)$.

To estimate $\tau_{w}$, let us consider a rod, which at time $t=0$ is oriented parallel to the wall, and located very close to the wall with $0<z \ll L / 2$. As the rod moves forward, it is reflected when it hits the wall, and is thereby constrained to the positive half-space $z>0$, see Fig. 3. This situation is very similar to a semi-flexible polymer, which is fixed at one end near the wall with tangent vector parallel to the wall; its bending rigidity $\kappa$ is determined by the persistence length, $\xi_{p}=\kappa / k_{B} T$. In this case, the distance of the polymers from the wall increases as $\langle z\rangle \sim\left(k_{B} T / \kappa\right)^{1 / 2} x^{3 / 2}$ and the orientation angle as $\langle\theta\rangle \sim\left(k_{B} T / \kappa\right)^{1 / 2} x^{1 / 2}$, where $x=v t$ is the distance traveled parallel to the wall $[17,18]$. The condition $\langle z\rangle=L / 2$ at $t=\tau_{w}$ then implies [15]

$$
\tau_{w} \sim \frac{1}{v}\left(L^{2} \xi_{p}\right)^{1 / 3} \sim\left(\frac{\eta}{k_{B} T}\right)^{1 / 3} L^{5 / 3} v^{-2 / 3}
$$

For the time $\tau_{b}$ for the rod to stay in the bulk fluid, we have to distinguish two regimes. In the ballistic regime, with $\xi_{p} \gg d$, the rod travels essentially on a straight line between the walls, see Fig. 3 . In this case, the bulk time is given by $\tau_{b} \sim$ $v^{-1} d / \sin (\theta)$, where $\theta$ is the angle of the rod with the surface when it leaves the wall layer of thickness $L / 2$. The polymer analogy explained above implies $\langle\theta\rangle \sim\left(L / \xi_{p}\right)^{1 / 3}$ for $\theta \ll 1$, so that $[15]$

$$
\tau_{b} \sim \frac{d}{v}\left(\frac{\xi_{p}}{L}\right)^{1 / 3} \sim\left(\frac{\eta}{k_{B} T}\right)^{1 / 3} d L^{2 / 3} v^{-2 / 3}
$$

Thus, the scaling arguments predict in the ballistic regime the probability

$$
p=L /\left(L+a_{B} d\right)
$$

to find the rod in the wall layers, with a constant $a_{B}$ which has to be determined numerically. Note that this expression is independent of the velocity $v$, because both 


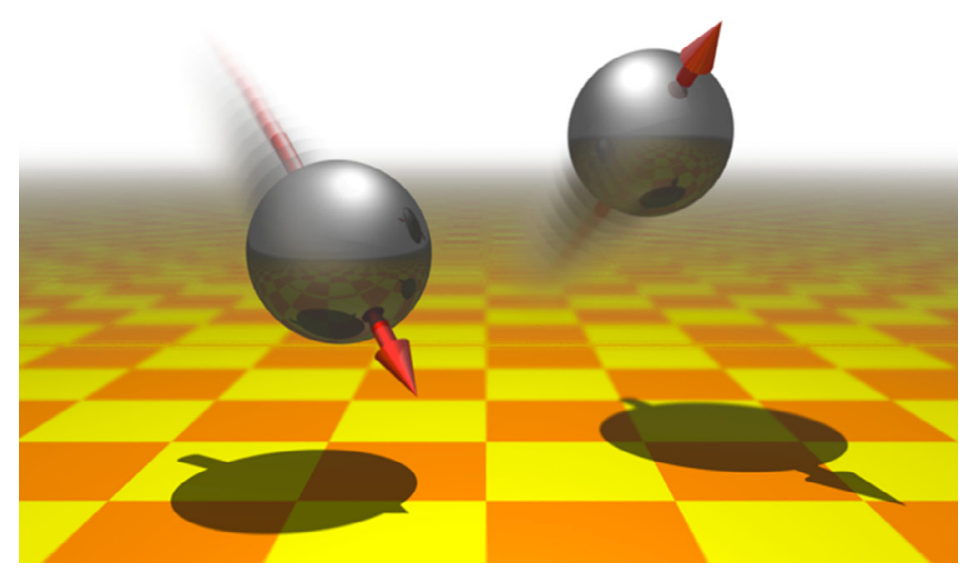

Fig. 4. Schematic of active Brownian spheres moving near a surface. From Ref. [20]. (This figure is subject to copyright protection and is not covered by a Creative Commons license.)

time scales $\tau_{w}$ and $\tau_{b}$ depend on $v$ in the same way. Thus, scaling theory explains the saturation of the excess surface density with increasing propelling force, as shown in Fig. 2(right).

For a system of many self-propelled Brownian rods between two walls in two dimensions, the rods moving along the walls in opposite directions block each other and lead to the formation of "hedgehog-like" clusters [19].

\subsection{Active Brownian spheres in confinement}

We have seen in the previous section that the alignment of rods with the surface plays an important role in their behaviour near surfaces. What happens if no such alignment mechanism is present? This question can be addressed by studying selfpropelled Brownian spheres, where the sphere orientation is completely independent of their spatial position, while the propulsion still drives the particles toward the walls, as shown schematically in Fig. 4.

Results of Brownian Dynamics simulations of active Brownian spheres in confinement between two hard walls are presented in Fig. 5. where the surface excess $s$ is shown as a function of the Péclet number $P e=v_{0} / \sqrt{D_{R} D_{T}}$ for different channel widths. These results demonstrate that the (normalized) probability density $\rho(\Delta z)$ to find a particle at a distance $\Delta z$ from the wall is strongly peaked close to the wall for $P e \gtrsim 5$. Note that $s$ is a monotonically increasing function of $P e$, and approaches unity for large $P e$ (complete adhesion).

The decoupling of the rotational degrees of freedom from translational motion allows for an analytic treatment via the Fokker-Planck equation [21]

$$
\partial_{t} \rho(z, \theta, t)=D_{R} \frac{1}{\sin (\theta)} \partial_{\theta}\left[\sin (\theta) \partial_{\theta} \rho(z, \theta, t)\right]-v_{0} \cos (\theta) \partial_{z} \rho(z, \theta, t)+D_{T} \partial_{z}^{2} \rho(z, \theta, t),
$$

where the angle $\theta=0$ corresponds to particles oriented in the positive $z$-direction. This equation already demonstrates the main origin of surface accumulation. The rotational diffusion is independent of the spatial position, but particles are driven to one of the chamber walls depending on their orientation. Thus particles oriented toward the top, accumulate at the top wall, those pointing down, accumulate at the bottom wall. Less particles remain in the center. Solutions for small Péclet number and 

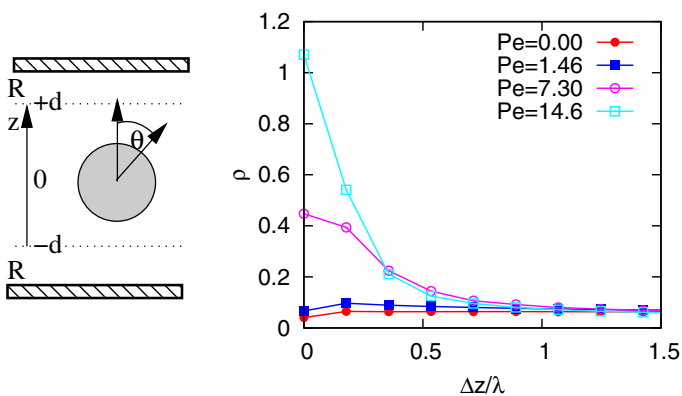

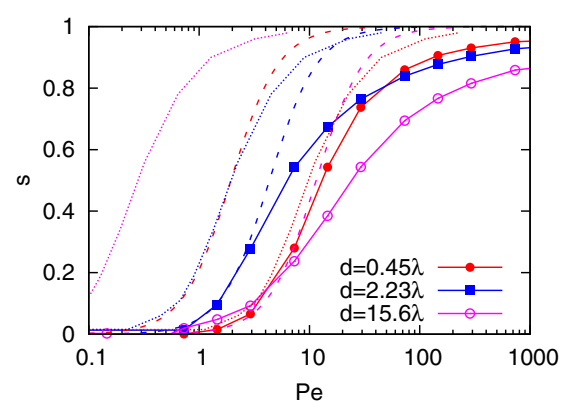

Fig. 5. (Left) A self-propelled Brownian sphere of radius $R$ is confined between two solid walls at $z= \pm(d+R)$. The orientation of the propulsion direction relative to the $z$-axis, is denoted by $\theta$. (Middle) Probability density $\rho(\Delta z)$ to find a particle at a distance $\Delta z$ from the wall. At zero Péclet number $P e=v_{0} / \sqrt{D_{R} D_{T}}$, the probability density is uniform beyond the short range of the repulsive wall. With increasing Péclet number particles accumulate near the wall. Results are shown for a system with wall separation $d / \lambda=15.6$, where $\lambda=\sqrt{D_{T} / D_{R}}$. (Right) Surface excess $s$ as a function of Péclet number $P e$ for various wall separations $d$, as indicated. Results from analytic calculation for very narrow channels (dotted lines) and for small Péclet numbers (dashed lines) are also shown. All analytic expression have no adjustable parameters. From Ref. [21]. (This figure is subject to copyright protection and is not covered by a Creative Commons license.)

narrow channels are depicted in Fig. 5, and work well within their respective limits [21]. For example for narrow channels the particle orientation can be considered a "slow variable", and the Fokker-Plank equation simplifies to a particle in an external field with a sedimentation velocity $v_{0} \cos (\theta)$. Thus the probability density $\rho(z, \theta)$ is found to be [21]

$$
\rho(z, \theta)=\frac{P e_{d} \rho_{0} \cos (\theta)}{\pi \sinh \left(P e_{d} \cos (\theta)\right)} \exp \left[\frac{v_{0} \cos (\theta) z}{d}\right]
$$

with $P e_{d}=v_{0} d / D$. Thus, the probability density of self-propelled spheres in narrow channels is also described by a Boltzmann-like distribution, as for passive Brownian particles in a gravitational field, but with an orientation-dependent field strength.

\subsection{Active Brownian dumbbells: Duality of shape asymmetry and wall curvature}

Most theoretical studies of simple model swimmers, both in bulk and in confinement, have considered so far cells with a symmetric body shape, in particular rods and spherical particles. In reality, however, cells usually do not exhibit such high symmetry and the stroke-averaged shape of sperm or Chlamydomonas algae rather resembles a forward or a backward swimming cone, respectively. This raises the question how a broken fore-aft symmetry of the particle shape alters the wall accumulation of cells.

In order to elucidate shape effects on effective adsorption, we neglect hydrodynamic interactions and study a generic model of an active Brownian dumbbell with unequal bead sizes [22]. Our Brownian dynamics simulation show that swimmers with a spermlike (polar) shape exhibit huge wall trapping times due to a non-vanishing component of the propulsion force directed toward the wall. This component of the propulsion force generates an effective barrier for reorientation, which has to be crossed due to (passive or active) orientational fluctuations before the swimmer can detach from the wall. Therefore, the trapping times increase exponentially 


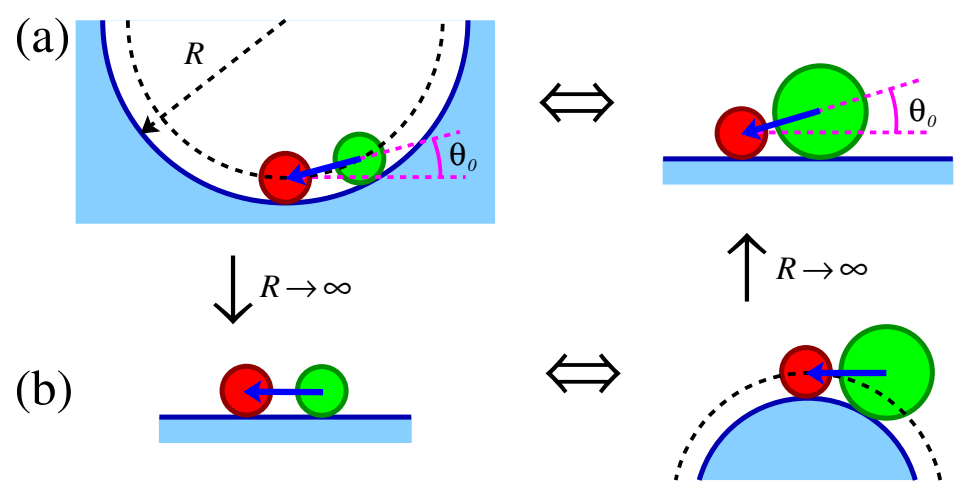

Fig. 6. (a) An apolar swimmer confined within a spherical cavity of radius $R$ is equivalent to a polar swimmer close to a flat wall - provided the angle $\theta_{0} \simeq-l / 2 R$ between the propulsion force of the apolar swimmer and the tangent plane of the cavity at the front bead, equals the asymmetry (opening angle) $\theta_{0} \simeq\left(a_{1}-a_{2}\right) / l$ of the polar particle, where $a_{1}$ and $a_{2}$ are the radii of the rear and front bead of the dumbbell, respectively, and $l$ is the bond length. (b) A polar particle near a convex boundary behaves like an apolar swimmer close to a flat wall if $\left(a_{1}-a_{2}\right) / l \simeq-l / 2 R$. From Ref. [22]. (This figure is subject to copyright protection and is not covered by a Creative Commons license.)

with the shape asymmetry (opening angle) $\theta_{0}$ and the propulsion strength (freeswimming velocity) $V$ and could, for realistic parameters of $\theta_{0}$ and $V$, exceed trapping times due to near-field hydrodynamic forces $[15,23,24]$. In contrast, microswimmers with Chlamydomonas-like (antipolar) shape behave similarly to symmetric rod-like particles.

Both in a natural environment and in microfluidic devices, microswimmers usually do not swim in straight, but rather in curved or branching microchannels. Therefore, the influence of surface curvature on accumulation of microswimmers is of great interest [25]. Based on the analysis of an asymmetric particle near a flat boundary, we can predict a direct duality relation between the effect of shape asymmetry and surface curvature on accumulation, as illustrated in Fig. 6. For example, a polar microswimmer close to a flat wall behaves similarly to an apolar particle near a concave surface (e.g., a cavity), see Fig. 6(a). In both cases, the velocity vector in the stable conformation forms an angle with the tangent plane to the wall at the front bead, so that the microswimmer points toward the wall and thus should have very long retention times [22]. Second, the force of a polar microswimmer towards the wall can be partially or fully compensated by a convex wall, i.e., for a microswimmer moving at the outer surface of a sphere of radius $R$, see Fig. 6(b). For full compensation, the same accumulation behaviour is predicted as for an apolar particle at a planar wall. Note that, in contrast, an apolar microswimmer would strongly scatter at a convex wall. Thus, shape polarity provides the possibility for microswimmers to move along curved surfaces. This is of high relevance for the design of microswimmers with controlled wall-adhesion properties.

\subsection{Run-and-tumble particles in confinement}

The simplification from a swimmer in confinement to a self propelled rod or sphere, can be taken even another step further. The run-and-tumble dynamics famous from E. coli chemotaxis [27] is in its idealized form of a random walker with finite step length well suited for mathematical treatment. 

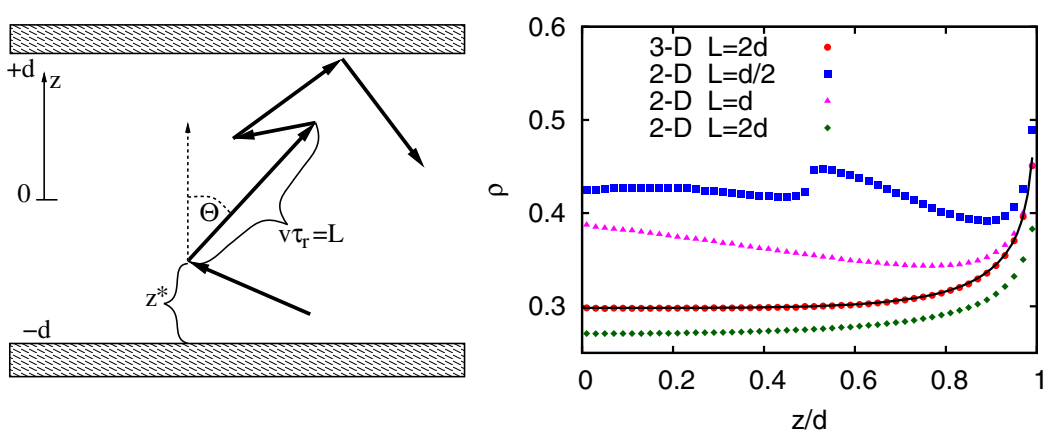

Fig. 7. (left) Schematic of run-and-tumble dynamics. A "run" of the particle with a velocity $v$ for a time $\tau_{r}$ is followed by a tumbling event, resulting in a new orientation $\theta$. The particle is confined between two parallel walls at $z= \pm d$. $z^{*}$ denotes the distance from the walls. (right) Particle density distribution $\rho(z)$ for various run lengths $L$. The solid line is the exact analytic solution for the particle density in three dimensions for run lengths larger than the channel width $2 d$. From Ref. [26]. (This figure is subject to copyright protection and is not covered by a Creative Commons license.)

During chemotaxis, E. coli swim in relative straight "runs" followed by "tumbling events" in which the bacterium reorients. The idealized version (see Fig. 7) we will study here, is similar to a random walk or Lévy flight. During the instantaneous tumbling event, the swimmer chooses a direction completely at random. The following straight run lasts either for a fixed time $\tau$, or is chosen from an exponential distribution with mean $\tau$.

In the bulk, far from surfaces, it has been shown that run-and-tumble dynamics and active Brownian motion are equivalent [28-30]. Even in slowly varying external potential $U(z)$, this equivalence holds. For example, the density distribution is found to be $[28]$

$$
\rho(z) \sim \exp [-\beta U(z)], \text { with } \beta=\frac{\mu \alpha}{v_{0}^{2}}=\frac{\mu}{D},
$$

where $\alpha$ is the tumbling rate, $\mu$ the particle mobility (the inverse friction coefficient), and $D=v_{0}^{2} /(\alpha d)$ the diffusivity (for spatial dimensionality $d$ ). This is exactly the same functional form of the Boltzmann distribution in thermal equilibrium, but now with an effective temperature $k_{B} T_{\text {eff }}=1 / \beta$. This effective temperature is typically much larger than the thermodynamic temperature for self-propelled particles. However, this equivalence breaks down in the vicinity of walls and surfaces [26].

For the study of run-and-tumble motion near walls and in confinement [26], we do not study the particle density, but the density of tumbling events $\Phi$. As tumbling events have no orientation, the tumbling density only depends on the distance to the surface $z$. We arrive thus at an one-dimensional problem. The particle orientation is effectively integrated out. The actual particle density can be obtained from the tumbling density by a convolution integral. Alternatively, it is also possible to work directly with the combined particle-orientation density [31]. While a bit more involved, it offers direct access to the mean particle orientation.

The Fokker-Planck equation for the tumbling density can be obtained from simple considerations. A particle tumbling at a distance to the surface $z$ will choose a random orientation and run straight for a distance $L=v_{0} \tau$. This results in a new position $a-$ $\Delta z$ an the process starts anew. If we call the probability of a certain $z$-displacement (or transfer function) $p(\Delta z)$, the tumbling event density evolves as [26]

$$
\phi\left(z, t+\tau_{r}\right)=\int_{-d}^{+d} \phi\left(z^{\prime}, t\right) p\left(z-z^{\prime}\right) d z^{\prime} .
$$


This however does not include the solid boundaries yet. In these dynamics however, this is rather simple. Particles just can not penetrate the wall, that is to say, if they hit the wall they remain there. This results in a delta peak of the tumbling density at the wall,

$$
\begin{array}{r}
\phi( \pm d, t)=0.5 \phi_{s}(t) \delta(z \pm d) \\
0.5 \phi_{s}\left(t+\tau_{r}\right)=\int_{-d}^{d} \phi\left(z^{\prime}, t\right) P\left(-z^{\prime}-d\right) d z^{\prime}
\end{array}
$$

where $\phi_{s}$ is the probability to find a tumbling event at the wall, and $P$ is the cumulative distribution function of $p$, i.e. $P(z)=\int_{-\infty}^{z} p\left(z^{\prime}\right) d z^{\prime}$.

Already from this simple intermediate result, we see that run-and-tumble particles will accumulate at the wall. In a rather thin channel it is quite simple: A particle tumbles at the wall. Half the time it just remains at the wall, in the rest of the cases, there is a high chance, that the run carries the particle straight over to the other wall. Thus most of the tumbling events happen at the wall.

To calculate this in more detail, one has to obtain the transfer function. The transfer function is the number of microstates (possible orientations), which are compatible with a certain displacement $\Delta z$ perpendicular to the wall. The number of microstates is proportional to the fraction of the surface area of the sphere of motion vectors, which generates a vertical displacement $\Delta z$. For example, in the case of constant run length $L$ in three dimensions, the transfer function is thus determined by [26]

$$
\begin{aligned}
p(z) d z & =\frac{1}{4 \pi L^{2}} \int_{0}^{2 \pi} d \varphi \int_{\arccos (z+d z)}^{\arccos (z)} d \theta L \sin (\theta) \\
& =-\left.\frac{1}{2 L} \cos (\theta)\right|_{\arccos (z+d z)} ^{\arccos (z)} \\
& =\frac{1}{2 L} d z
\end{aligned}
$$

for $|z|<L$. Thus, the transition probability for vertical displacements is uniform in three dimensions. In two dimensions, an equivalent derivation shows that the transfer function diverges instead at $\pm L$

$$
p_{(2, c)}(z)=\frac{1}{\pi L \sqrt{1-(z / L)^{2}}} \Theta(L-z) \Theta(L+z) .
$$

The discontinuity at $\Delta z= \pm L$ leads to peaks and interference patterns in the tumbling density (see Fig. 8). The simplicity of the 3D transfer function allows for an analytic solution for narrow channels with $2 d<L$,

$$
\phi_{(3, c)}(z)=\frac{1}{2 L}+\frac{1-d / L}{2}[\delta(z-d)+\delta(z+d)]
$$

The solution for two dimensions or larger channel widths can only be obtained with certain simplifications. See Ref. [26] for more details.

In the case of more general run length distributions, one simply uses the conditional probability to obtain the transfer function:

$$
p_{(n, l e n)}(z)=\int_{z}^{\infty} P_{(n, c)}\left(z \mid L^{\prime}\right) p_{l e n}\left(L^{\prime}\right) d L^{\prime}
$$



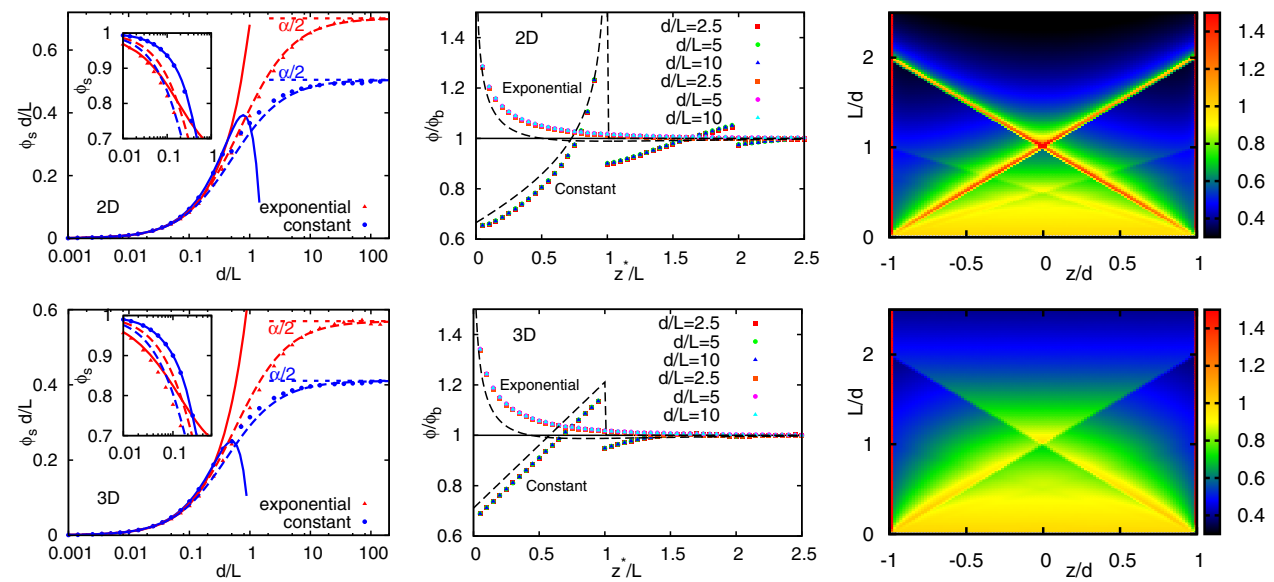

Fig. 8. Tumbling density profiles $\phi(z)$ for (top) two and (bottom) three dimensions. (left) Scaled surface density $\phi_{s} d / L$ as a function of the ratio $d / L$ of channel width and run length. Solid lines are analytical approximations for narrow channels (see text), dashed lines are fits to a large-channel approximation. Note that for $d \gg L,\left(\phi_{s} d / L\right)$ approaches $\alpha / 2$. (center) Scaled tumbling density $\phi\left(z^{*}\right) / \phi_{b}$ as a function of the scaled distance $z^{*} / L=(z+d) / L$ from the wall. Dashed lines show the approximation $\phi_{1}\left(z^{*}\right)$, which is obtained from a first-order iteration of Eq. (8) (see text). (right) Density of tumbling events $\phi(z / L)$ inside the channel for various (constant) run lengths. For $2 d \geq L$, the presence of the two walls induces strong density modulations. From Ref. [26]. (This figure is subject to copyright protection and is not covered by a Creative Commons license.)

with the run-length distribution $p_{l e n}\left(L^{\prime}\right)$ For exponential run-length distributions, the broadness of the run length distribution destroys the peaks and valleys away from the wall, and only an increased density at the wall is left (see Fig. 8).

These calculations are, however, only valid for an idealized run-and-tumble dynamics. For the description of the behaviour of real bacteria, a certain persistence in the tumbling events [32] and the influence of walls on tumbling frequency [33] probably has to be taken into account, and may significantly affect the results. Thus, it remains challenging to understand and predict the dynamics of real E. coli in confinement.

\section{Hydrodynamic interactions of microswimmers with surfaces}

\subsection{Microswimmer hydrodynamics}

Most microswimmers move autonomously, with no external force applied, and hence the total interaction force of the swimmer on the fluid, and vice versa, vanishes. In the simplest case, which actually applies to many microswimmers like bacteria, spermatozoa, or algae, the far-field hydrodynamics (at distances from the swimmer much larger than its size) can be well described by a force dipole [34,35]. For a detailed description and discussion of the dipole flow field see minireview [36] by R.G. Winkler in this issue. This approximation has been confirmed experimentally for E. coli [37]. Two classes of such dipole swimmers can be distinguished, as shown schematically in Fig. 9. If the swimmer has its motor in the back, and the passive body drags along the surrounding fluid in front, the characteristic flow field of a "pusher" emerges, see Fig. 9(a). Similarly, if the swimmer has its motor in the front, and the passive body drags along the surrounding fluid behind, the characteristic flow field of a "puller" develops, see Fig. 9(b). Thus, sperm, E. coli and salmonella are pushers, because they 


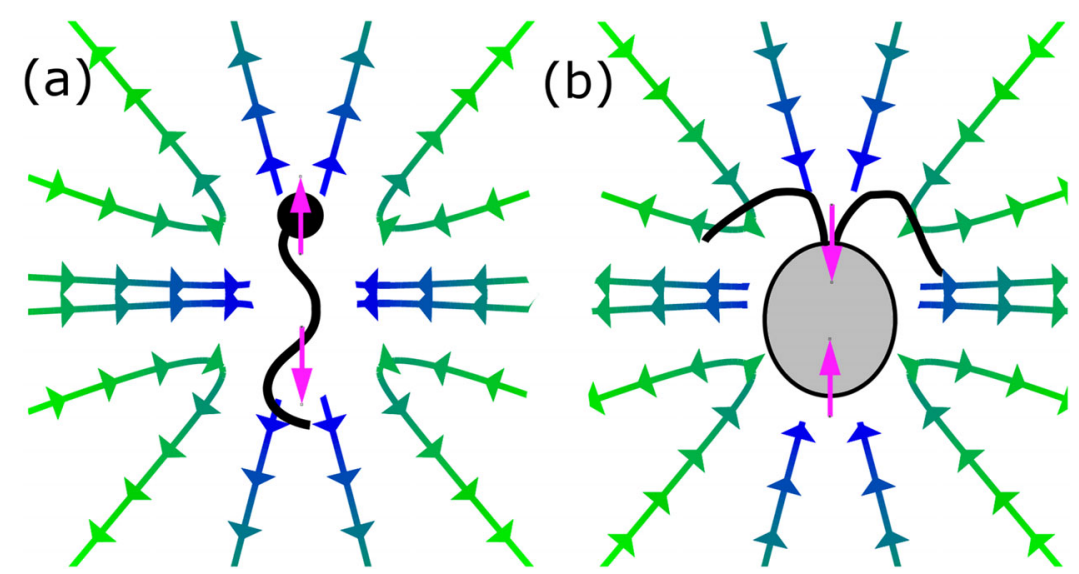

Fig. 9. Schematics of the flow field of dipole swimmers, (a) pusher (represented by a sperm cell) and (b) puller (represented by the green alga C. reinhardtii). From Ref. [5]. (This figure is subject to copyright protection and is not covered by a Creative Commons license.)

have active propelling flagella in the rear, and a passive head in the front (for details, see minireview [38] by L. Alvarez \& U.B. Kaupp for sperm). In contrast, the green alga C. reinhardtii is a puller, because the two flagella in the front push the fluid backwards, while the body in the rear remains passive, like for a human breast-stroke swimmer (for details, see minireview [39] by M. Polin in this issue).

The magnitude of the flow field is measured by dipole strength $P$, which is given by the product of dipole length and its force (positive for pushers and negative for pullers). It is important to notice that the flow fields of pushers and pullers are identical, but with opposite flow directions. This has important consequences for the interactions between microswimmers, and of microswimmers with walls, as will be explained below. The dipole force of sperm can be estimated from the friction force of the whole body, which balances the pushing force of the tail, and is thus proportional to $\eta v L$, where $v$ is the swimming velocity and $L$ the sperm length. The dipole strength therefore scales as

$$
P \sim \eta v L^{2} .
$$

A first idea about the effect of hydrodynamic interactions on the dynamics of microswimmers near surfaces can be obtained from this far-field approximation, which applies when the size of the swimmer is much smaller than its distance from the surface. In this limit, analytical expressions for the torque on a swimmer and its drag velocity towards the surface can be obtained [40]. For a swimmer with an orientation angle $\theta$ of the dipole with respect to the vertical $(z)$ direction (i.e. $\theta=90^{\circ}$ when the swimmer moves parallel to the wall), the induced velocity at a distance $z$ away from the no-slip wall is given by [40]

$$
u_{z}(\theta, z)=-\frac{3 P}{64 \pi \eta z^{2}}\left(1-3 \cos ^{2} \theta\right) .
$$

Equation (15) allows several interesting predictions. For the discussion, we focus on the case of pushers (like sperm). First, the hydrodynamic interaction is attractive for pushers oriented nearly parallel to the wall (with $\theta$ near $90^{\circ}$ ), but becomes repulsive for pushers oriented nearly perpendicular to the wall (with $\theta$ near $0^{\circ}$ ). Second, the hydrodynamic interaction is long-ranged, with a $1 / z^{2}$ decay with increasing 


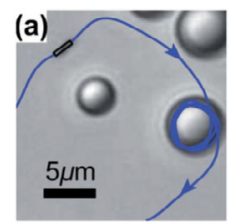

(b)

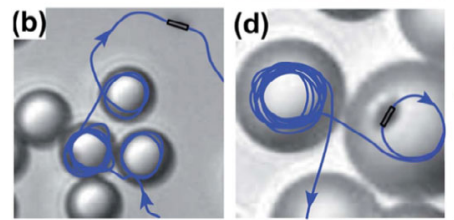

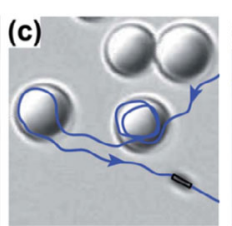

(d)
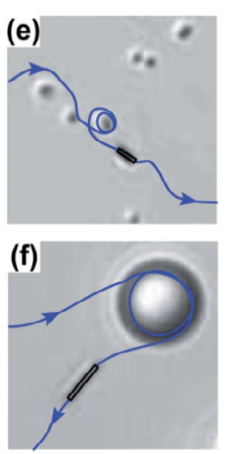

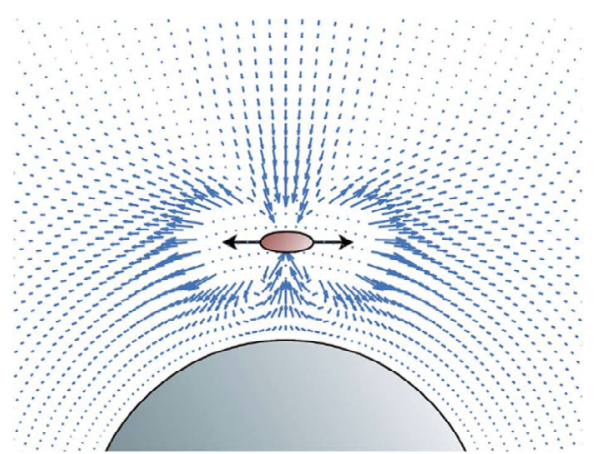

Fig. 10. Trapping of microswimmers by spherical colloids due to hydrodynamic interactions. (a) Experiments with self propelled Pt-Au rods in $\mathrm{H}_{2} \mathrm{O}_{2}$ solution interacting with colloidal obstacles [41]. (b) Hydrodynamic interactions trap a force dipole on a spherical colloid. The flow field looks similar to the one of a dipole next to a planar wall, and indeed the hydrodynamic interactions are very similar [23]. Reproduced with permission from Refs. [41] and [23]. (This figure is subject to copyright protection and is not covered by a Creative Commons license.)

distance from the surface. The insertion of the order-of-magnitude estimate for the dipole strength of Eq. (14) shows that the drift towards the surface is proportional to the swimmer velocity and to $(z / L)^{2}$. Finally, the hydrodynamic interaction not only generates a force on the swimmer, but also a torque [40]. It turns out that this torque always acts to align pushers parallel to the surface - the orientation in which the hydrodynamic force is attractive.

This hydrodynamic torque and attraction of microswimmers work similarly close to curved surfaces [23]. It was found experimentally that chemically-powered diffusophoretic rod-like microswimmers are captured by larger colloids (see Fig. 10). The moving rods travel through the fluid, and occasionally encounter suspended spherical colloids. If the encounter is close enough, they typically circle around it a few times, before departing again. Analytical and numerical calculations [23] show that a force dipole creates a hydrodynamic torque orienting the swimmer tangentially to a spherical surface, and generates an attractive force, similarly as for planar walls. However, theses forces now depend on the sphere radius, and, more importantly are counterbalanced by a simple geometrical effect - as a particle traveling tangentially to the surface, will quickly move away, and also point away, from the surface. This balance between hydrodynamic attraction, and "geometric repulsion" leads to a critical minimal colloid size which is necessary to to trap microswimmers by hydrodynamic dipole interactions alone. The calculations show that a microswimmer approaching a smaller spherical colloid is simply scattered [23]. Contrary to the trapping by planar walls, the finite size of the colloid results in a very small basin of attraction (see Fig. 11), i.e. a particle moving parallel to the colloids surface needs to be very close to the colloid itself in order to get trapped. Interestingly, the size of the attractive region does not scale linear with the colloid size, but with a sublinear power law $A^{1 / 5}$ [23].

However, in the experiments, the self-propelled rods also circle around very small colloids [41] (see Fig. 10). It remains an open question whether this behavior is due to the hydrodynamic near-field interactions, steric interactions, or the disturbance of the concentration gradient around the swimmer. Furthermore, it would be interesting to study the combined effect of shape asymmetry (as discussed in Sect. 2.3) and hydrodynamic attraction on the behavior of microswimmers near curved surfaces.

Similarly, the interaction of two microswimmers at long distances is determined by their dipole flow fields. The dipole approximation again predicts that the 

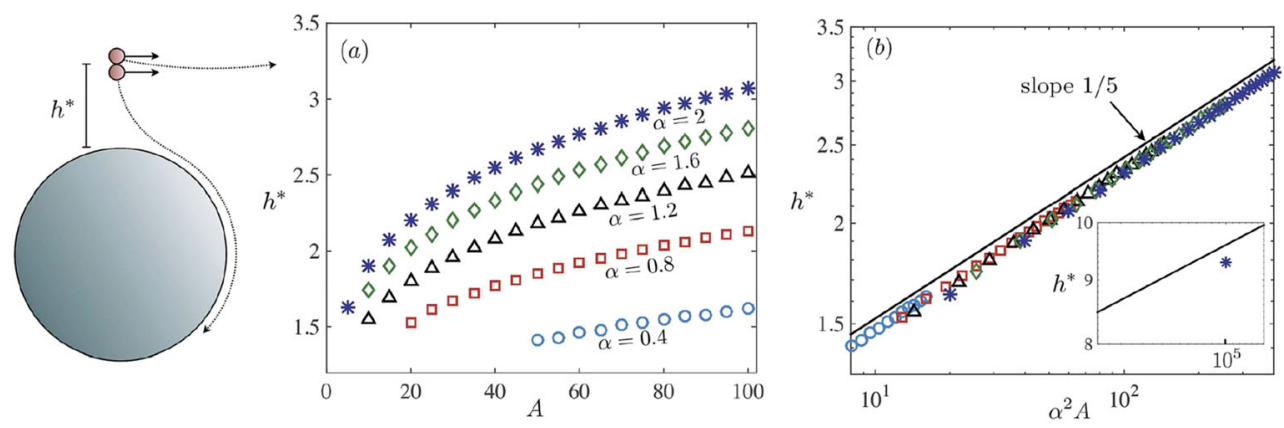

Fig. 11. Basin of attraction for trapping of microswimmers by spherical colloids of radius $A$, due to hydrodynamic interactions. For a spherical swimmer placed initially parallel to the surface, $h^{*}$ denotes the critical initial distance from the colloid above which the particle escapes, and below which entrapment ensues. (a) The critical initial distance for a selection of dimensionless dipole strengths $\alpha$, shown where the colloid size is larger than the critical size for entrapment. (b) The curve collapses upon plotting against the squared dimensionless dipole strength times the colloids radius, $\alpha^{2} A$, to a power law scaling with exponent $1 / 5$. The solid line is the prediction from analytic calculations (inset). Reproduced with permission from Ref. [23]. (This figure is subject to copyright protection and is not covered by a Creative Commons license.)

interactions of pushers and pullers have opposite sign, because their dipole strengths $P$ have opposite signs. This prediction does not only hold in the far field, but - qualitatively - also when two microswimmers approach each other closely. This behaviour can be studied explicitly by considering the flow fields of two parallel-swimming self-propelled spheres [42] at smaller distances. A model of a spherical particle with a fixed (cylindrically symmetric) fluid-velocity pattern on its surface is called a "squirmer" [43]. Pushers can be described in this model by having a fast backward surface velocity on the trailing hemisphere, and a slow or vanishing forward velocity on the foreward facing hemisphere. The results of mesoscale hydrodynamics simulations (for details about such simulation techniques, see minireview [36] by R.G. Winkler in this issue) of two squirmers shown in Fig. 12 demonstrate that for pushers, the fast backward flow velocity in the rear part extracts fluid from the gap between the swimmers, and thereby induces attraction (Fig. 12a); in contrast, for pullers, the fast backward flow velocity in the front part injects fluid into the gap, and thereby induces repulsion (Fig. 12b) [42]. At the small squirmer separation show in Fig. 12, the interaction is dominated by the hydrodynamic near-field, and the far-field approximation does not allow quantitative predictions anymore. In this case, the details of the specific swimmer at hand become important. In the following, we thus study exemplarily two model swimmers: Sperm cells, that swim by the snake-like motion of their tail, and bacteria, that propel by a rotating cork-skrew shaped filament.

The squirmer model has also been employed to study the effects of the hydrodynamic near field on the swimming motion near surfaces [44,45].

\subsection{Sperm motion near surfaces}

A second contribution comes from the sperms asymmetry. The tails beat envelope is much bigger than the head, creating an effective triangle like shape. This front-back asymmetry can create giant surface accumulation [22]. Indeed, simulations suggest [46] that the beat pattern creates a sideways outflow at the tail. 

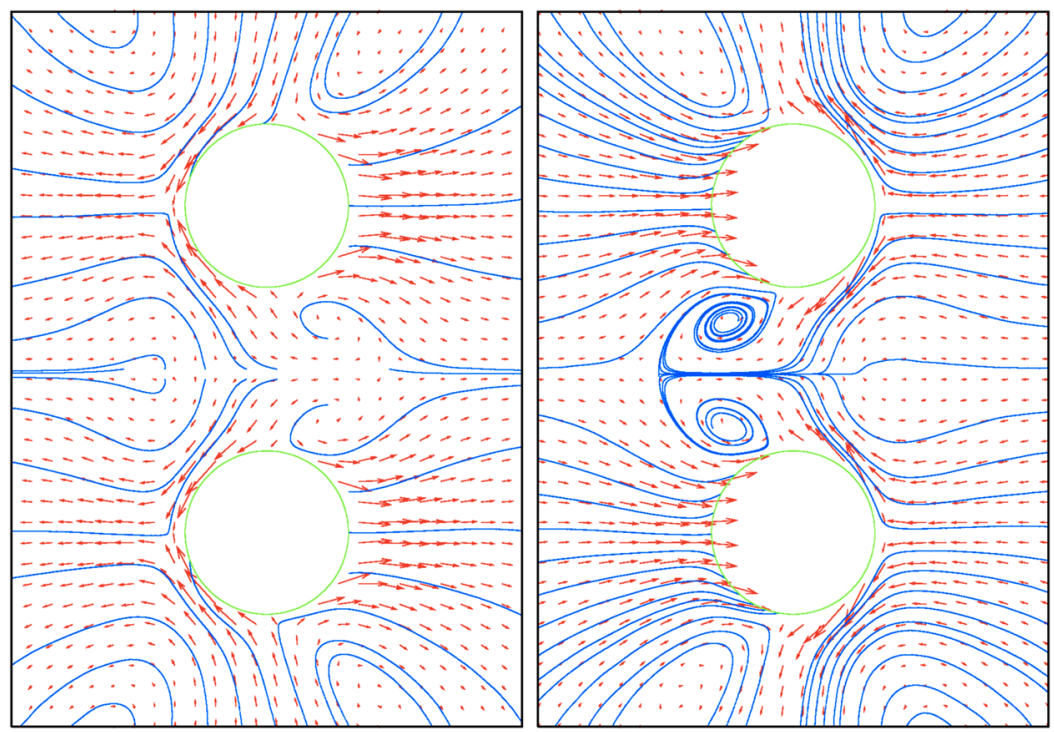

Fig. 12. Velocity fields for a pair of squirmers with fixed distance and fixed parallel orientation, for (left) pusher and (right) puller, with Péclet number Pe $=1155$. Swimmers move to the right. Streamlines serve as a guide to the eye. Only a fraction of the simulation box is shown. (Due to the finite resolution of the measured velocity field, some streamlines (unphysically) end on the squirmers' surfaces.) From Ref. [42]. (This figure is subject to copyright protection and is not covered by a Creative Commons license.)

This creates a repulsion of the tail, resulting in a torque, leading to the sperm cell pointing towards the wall. It was later confirmed experimentally that indeed sperm seem to be swimming at the wall, with a small but finite angle pointing against the surface [11].

Thus the adhesion of sperm cells to surfaces is a combination of several different contributions, and it is hard to identify which is the dominant one. Furthermore, sperm do not swim in straight lines, but typically on preferably right handed circles (see Fig. 14). Simulations show that a asymmetry in the head can be sufficient to explain not only circular motion, but also the preferred handedness. One can speculate that sperm use this kind of asymmetry to navigate towards the egg in drifting circles [47] or helices [48].

Generic models can help to understand how different effects contribute to the swimmer-surface interaction. However, each microswimmer has its own combination of contributions, and often additional non universal effects matter. Sperm cells for example swim very close to nearby walls. Indeed the distance to the wall is much smaller than the sperms length, rendering the multipole expansion invalid. Nevertheless Sperm-cell-like beating close to a wall creates a flow field, very similar to that of a pusher (see Fig. 13).

The circular motion of sperm near surfaces also gives rise to an interesting collective behaviour at higher densities - where sperm form quite regular arrays of vortices in which several sperm swim together in tight circles [49,50] - and in shear flow where sperm align against the flow direction and can swim upstream with a fixed deviation angle (rheotaxis) [51-53]. A discussion of more generic aspects of circle swimmers is given in the minireview [54] by $\mathrm{H}$. Löwen in this issue; an overview of the behaviour of microswimmers in external (flow) fields is provided in the minireview [55] by H. Stark. 

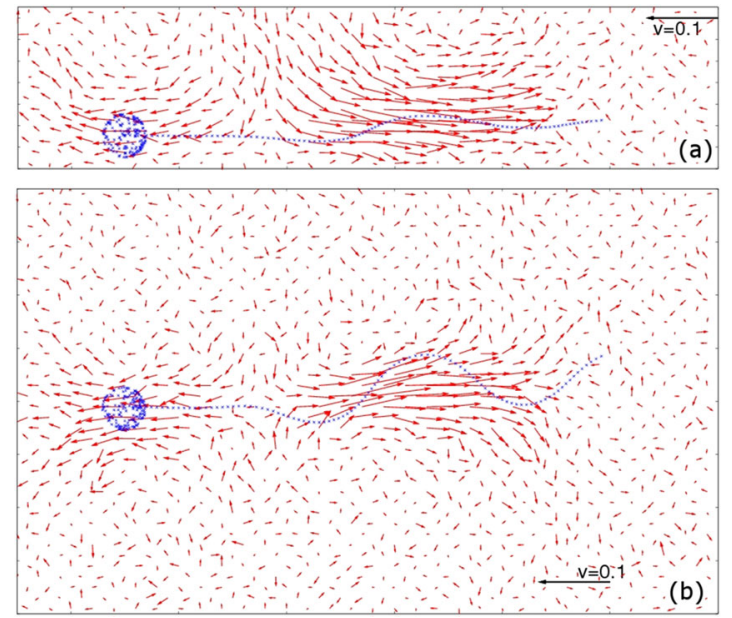

Fig. 13. Averaged flow field in the vicinity of a sperm cell adhering to a wall. (a) Plane perpendicular to the wall, and (b) plane parallel to the wall, with both planes containing the average sperm shape. A snapshot of a sperm is superimposed. The flow field generated by the beating tail is directed away from the sperm along their swimming direction and towards the sperm along its side. From Ref. [46]. (This figure is subject to copyright protection and is not covered by a Creative Commons license.)

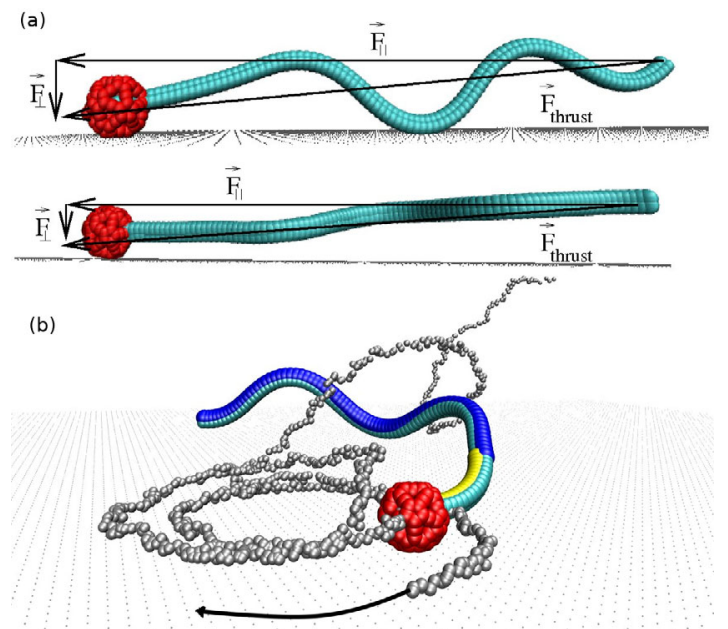

Fig. 14. (a) Sketch of forces responsible for the adhesion of sperm at a surface. Top: Without hydrodynamic interactions, the beating-plane of adhering sperm is oriented perpendicular to the surface. Bottom: With hydrodynamic interactions, the beating-plane is oriented parallel to the surface. (b) Sperm (model with curved and elastically deformable midpiece) with large preferred curvature $\left(c_{0}^{(m)} L_{m}=1\right)$ at a surface. The head touches the wall and blocks further rotation. The beating plane is approximately perpendicular to the surface. From Ref. [46]. (This figure is subject to copyright protection and is not covered by a Creative Commons license.)

\subsection{Bacterial motion close to homogeneous and striped surfaces}

Most bacteria exploit helical filaments for propulsion, driven by rotary motors located in their cell membrane. Prominent examples of peritrichous bacteria, which possess numerous flagella, are Escherichia coli, Salmonella typhimurium, Rhizobium lupini, 
(a)

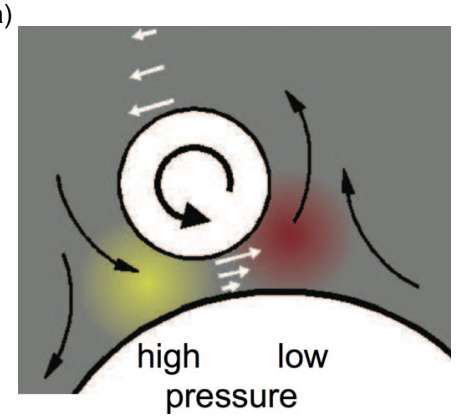

(b)

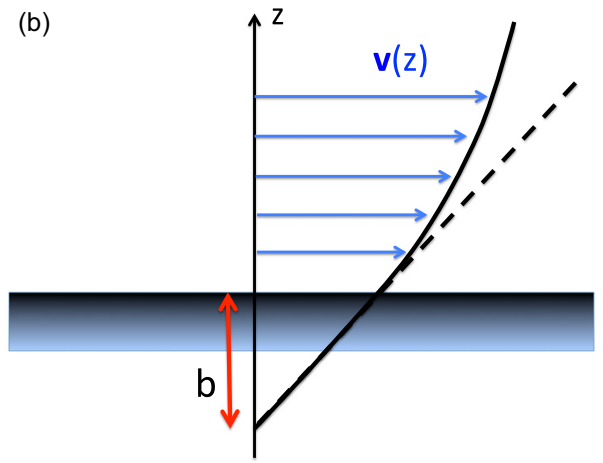

Fig. 15. (a) Flow and pressure fields, which develop for a rotating body near a planar or curved wall. From Ref. [62]. (b) Definition of the Navier slip length $b$ for simple shear flow near a solid wall. (This figure is subject to copyright protection and is not covered by a Creative Commons license.)

or Proteus mirabilis to name just a few. In a bulk fluid, the bacteria move in a straight manner (run), with all flagella forming a bundle, interrupted by abrupt changes of the swimming direction (tumble) induced by disintegration of the bundle [27], as mentioned above. The presence of a surface drastically alters the swimming behaviour. For instance, the non-tumbling mutant of E. coli swims in a clockwise (CW) circular trajectory close to a solid boundary $[4,56]$ and a counterclockwise $(\mathrm{CCW})$ trajectory close to a liquid-air interface [56-58]. Hence, bacteria are able to "sense" the properties of a nearby surface, an aspect of great importance for surface selection and attachment in the early stages of biofilm formation or infection $[4,59,60]$. Also, a theoretical understanding of hydrodynamic interactions between swimming bacteria and surfaces not only sheds light on selective surface attachment, but opens an avenue for the design of microfluidic devices to control and guide bacterial motion [3] for separation, trapping, stirring, etc. [61].

The swimming behaviour of bacteria near surfaces is governed by hydrodynamic forces [5,34] and, hence, the $\mathrm{CW}$ and $\mathrm{CCW}$ circular trajectories of $E$. coli have to be explained in terms of hydrodynamic interactions. The basic physical mechanism is as follows. The rotary motors generate rotations of the flagellar bundle and of the cell body in opposite directions, such that the whole swimmer experience no net (external) torque. Now, a rotating body near a wall, with the axis of rotation parallel to the wall, experiences two forces induced by the flow, as illustrated in Fig. 15a. The first force is due to the velocity gradient of the fluid in the gap between the wall and the rotating body; it pushes the body in the "rolling" direction. The second force is due to a pressure difference between the two sides of the cylinder, which arises from the compression of the fluid into the gap on one side, and the decompression on the other side; it pushes the body against the rolling direction. The actual motion depends on the balance between these two forces. For a no-slip wall, they exactly cancel for an infinitely long cylinder, while the shear force dominates for a sphere (and a short spherocylinder) and generates a motion in the rolling direction. This implies that in addition to the propulsive forward force - the bacterial body experiences a force to the right, the flagellar bundle to the left (due to the opposite directions of rotation), which results in a CW circling motion close to the wall.

Fluid slip on a surface at $z=0$ is characterized by the slip length $b$, which is defined by the boundary condition

$$
\mathbf{v}_{s}=b \frac{\partial \mathbf{v}}{\partial z}
$$


a

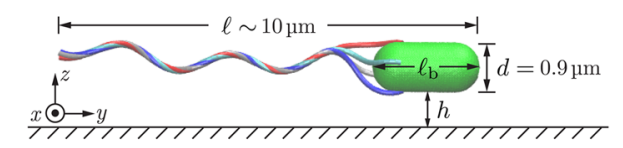

c

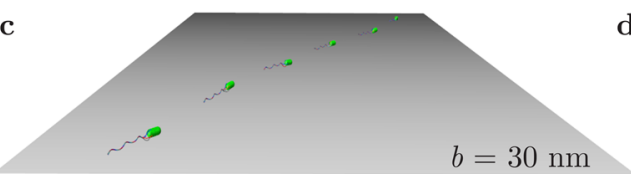

b

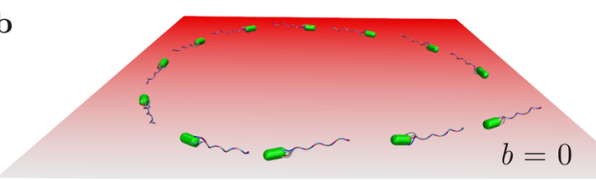

d

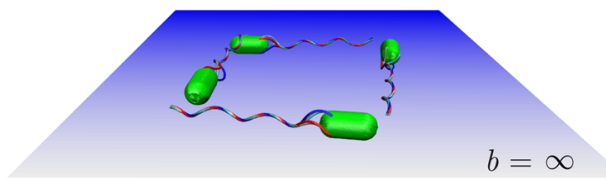

Fig. 16. Swimming bacteria sense the slip of its nearby surface. (a) The model bacterium of length $\ell$ consists of a spherocylindrical body of length $\ell_{b}$ and diameter $d$ and four helical flagella each turned by a motor torque. The bacterial geometry and flagellar properties are in agreement with experiments of $E$. coli. The body and the flagellar bundle counter rotate. $h$ is the gap width between the body and the surface. (b) CW, (c) noisy straight, and (d) CCW trajectories from hydrodynamic simulations of a bacterium swimming near homogeneous surfaces with different slip lengths $b$, as indicated. From Ref. [63]. (This figure is subject to copyright protection and is not covered by a Creative Commons license.)

for the fluid flow field $\mathbf{v}(\mathbf{r}, t)$, where $\mathbf{v}_{s}$ is the fluid velocity at the surface, as illustrated in Fig. 15b. Now, a finite slip length $b$ implies that the fluid velocity gradient in the gap between the rotating body and the wall is reduced, so that the corresponding force contribution is reduced compared to the no-slip case. For a perfect-slip wall, with $b=\infty$, the pressure contribution dominates, and the bacterium performs a CCW motion.

The dependence of the motion on the slip length can be studied numerically by a bacterium model displayed in Fig. 16a. This model consists of a spherocylindrical body and several helical flagella, which are driven by a motor torque at their anchoring points in the cell wall $[63,64]$. The bacterium model is then embedded in a particle-based mesoscopic solvent to describe hydrodynamic flows and interactions, as explained in more detail in the minireview [36] by R.G. Winkler in this issue. Simulations with this model reproduce the two limiting cases of slip lengths very well, see Fig. 16b,d. Obviously, there should be an intermediate value of the slip length, where the bacterium swims on a straight line, which is predicted from the simulations for $E$. coli to occur for $b=30 \mathrm{~nm}$. This is an interesting result, because it shows that the bacterium motion is sensitive to changes in slip length on the tens of nanometer scale. Swimming bacteria could therefore act as slip-length sensor on this scale of slip lengths.

The possibility of manipulating the bacterial motion by surface modification can be taken one step further by using structured walls [63]. By using striped surfaces, for which neighboring stripes induce an opposite sense of circular motion, bacteria can be made to follow stripe boundaries in a snaking-like motion, as they are always directed back to the stripe boundary by the alternating $\mathrm{CW}$ and $\mathrm{CCW}$ motions. Of course, this snaking motion is only possible if the stripe width $L$ is large enough that the bacterium does not hit a second stripe boundary before being able to return to the first. Therefore, in the case that $L$ is smaller than the circle radius $R$, the bacterial motion is hardly affected by the surface structure. This is illustrated in Fig. 17a,b. As a results, the diffusional motion depends strongly on the ratio $L / R$, and becomes highly anisotropic for $L / R>1$, as shown in Fig. 17c. Striped surfaces can therefore be employed to separate bacteria with different trajectory radii! 

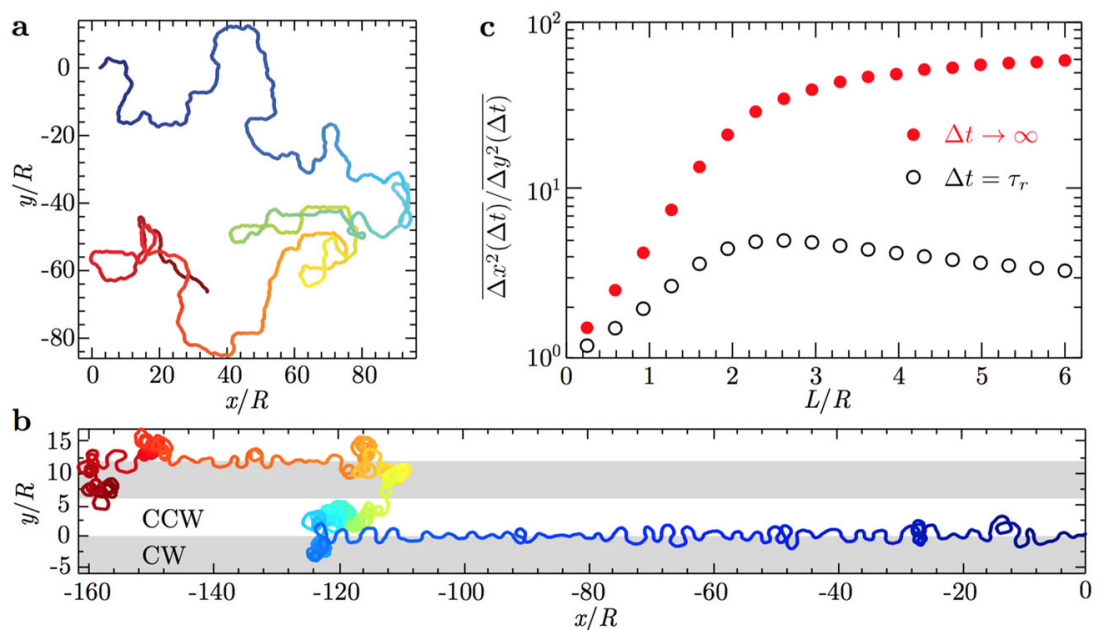

Fig. 17. Active Brownian rod swimming on a "symmetric" striped surface with curvature radii equal in magnitude but opposite in sign. Trajectories for (a) narrow $(L / R=0.25)$ and (b) broad $(L / R=6)$ stripes from simulations with starting points indicated in dark blue. (c) The ratio of mean square displacement (MSD) parallel (x) and perpendicular (y) to the stripes as a function of stripe width $L$ for $\Delta t$ equal the rotational diffusion time $\tau_{r}$ and for $\Delta t \rightarrow \infty$. From Ref. [63]. (This figure is subject to copyright protection and is not covered by a Creative Commons license.)

\section{Outlook}

Surfaces and confinement provide a multitude of possibilities to control the behaviour of microswimmers. A detailed understanding of the mechanisms at play allows for the design of microfluidic devices tailored for specific tasks. Effects like surface adhesion or circular swimming can be exploited to sort microswimmers, perform surface analysis, or concentrate microswimmers and perform tasks on other particles. Discoveries of new surface effects will enable us to conceive novel applications of confined microswimmers for specific purposes.

While the simplest of the fundamental questions have been answered recently, many more questions remain. How do hydrodynamic interactions look like for more complex confining geometries, i.e. beyond the infinite planar wall? What is the effect of curvature of surfaces and microchannels? What is the role of (active) noise in competition with hydrodynamics [24]? How is the swimming motion near surfaces affected by the viscoelasticity of the fluid [65]? What is the swim pressure exerted on a wall by a microswimmer suspension, and how does it depend on the swimmer shape, the wall interaction, and hydroynamics $[22,31,66-70]$ ? How does a surface influence the collective behaviour? These, and many more, questions will have to be addressed, before we can truly say we understand the interactions of microswimmers in confinement.

Stimulating discussions with Roland G. Winkler and Adam Wysocki are gratefully acknowledged. The long-standing collaboration with Louis Alvarez and Benjamin Kaupp has been essential for all work on sperm cells. This work was supported by the DFG priority program SPP 1726 on "Microswimmers - from Single Particle Motion to Collective Behaviour" We also gratefully acknowledge a CPU-time grant by the Jülich Supercomputing Center. 


\section{References}

1. L. Rothschild, Nature 198, 1221 (1963)

2. B.M. Friedrich, I.H. Riedel-Kruse, J. Howard, F. Jülicher, J. Exp. Biol. 213, 1226 (2010)

3. W.R. Diluzio, L. Turner, M. Mayer, P. Garstecki, D.B. Weibel, H.C. Berg, G.M. Whitesides, Nature 435, 1271 (2005)

4. E. Lauga, W.R. DiLuzio, G.M. Whitesides, H.A. Stone, Biophys. J. 90, 400 (2006)

5. J. Elgeti, R.G. Winkler, G. Gompper, Rep. Prog. Phys. 78, 056601 (2015)

6. G.A. Ozin, I. Manners, S. Fournier-Bidoz, A. Arsenault, Adv. Mater. 17, 3011 (2005)

7. S. Sengupta, M.E. Ibele, A. Sen, Angew. Chem. Int. Ed. 51, 8434 (2012)

8. G. Gompper, Spektrum der Wissenschaft Heft 4, 84 (2015)

9. H.P. Zhang, A. Be'er, E.-L. Florin, H.L. Swinney, Proc. Natl. Acad. Sci. USA 107, 13626 (2010)

10. S.E. Hulme, W.R. DiLuzio, S.S. Shevkoplyas, L. Turner, M. Mayer, H.C. Berg, G.M. Whitesides, Lab Chip 8, 1888 (2008)

11. P. Denissenko, V. Kantsler, D.J. Smith, J. Kirkman-Brown, Proc. Natl. Acad. Sci. USA 109, 8007 (2012)

12. M.C. Marchetti, Y. Fily, S. Henkes, A. Patch, D. Yllanes, Curr. Opin. Colloid Interface Sci. 21, 34 (2016)

13. T. Speck, Eur. Phys. J. Special Topics 225, 2287 (2016)

14. F. Peruani, Eur. Phys. J. Special Topics 225, 3001 (2016)

15. J. Elgeti, G. Gompper, EPL 85, 38002 (2009)

16. G. Li, J.X. Tang, Phys. Rev. Lett. 103, 078101 (2009)

17. A.C. Maggs, D.A. Huse, S. Leibler, Europhys. Lett. 8, 615 (1989)

18. T.W. Burkhardt, J. Stat. Mech. p. P07004 (2007)

19. H.H. Wensink H. Löwen, Phys. Rev. E 78, 031409 (2008)

20. J. Elgeti, R.G. Winkler, G. Gompper, SoftComp Newsletter 12, (2015), http://www. eu-softcomp.net.

21. J. Elgeti G. Gompper, EPL 101, 48003 (2013)

22. A. Wysocki, J. Elgeti, G. Gompper, Phys. Rev. E 91, 050302(R) (2015)

23. S.E. Spagnolie, G.R. Moreno-Flores, D. Bartolo, E. Lauga, Soft Matter 11, 3396 (2015)

24. K. Schaar, A. Zöttl, H. Stark, Phys. Rev. Lett. 115, 038101 (2015)

25. Y. Fily, A. Baskaran, M.F. Hagan, Soft Matter 10, 5609 (2014)

26. J. Elgeti, G. Gompper, EPL 109, 58003 (2015)

27. H.C. Berg, E. coli in Motion (Springer, New York, 2004)

28. J. Tailleur, M.E. Cates, EPL 86, 60002 (2009)

29. M.E. Cates, J. Tailleur, EPL 101, 20010 (2013)

30. A.P. Solon, M.E. Cates, J. Tailleur, Eur. Phys. J. Special Topics 224, 1231 (2015)

31. B. Ezhilan, R. Alonso-Matilla, D. Saintillan, J. Fluid Mech. 781, R4 (2015)

32. H.C. Berg, D.A. Brown, Nature 239, 500 (1972)

33. M. Molaei, M. Barry, R. Stocker, J. Sheng, Phys. Rev. Lett. 113, 068103 (2014)

34. E. Lauga, T.R. Powers, Rep. Prog. Phys. 72, 096601 (2009)

35. T. Ishikawa, J.R. Soc. Interface 6, 815 (2009)

36. R.G. Winkler, Eur. Phys. J. Special Topics 225, 2079 (2016)

37. K. Drescher, J. Dunkel, L.H. Cisneros, S. Ganguly, R.E. Goldstein, Proc. Natl. Acad. Sci. USA 108, 10940 (2011)

38. U.B. Kaupp, L. Alvarez, Eur. Phys. J. Special Topics 225, 2119 (2016)

39. R. Jeanneret, M. Contino, M. Polin, Eur. Phys. J. Special Topics 225, 2141 (2016)

40. A.P. Berke, L. Turner, H.C. Berg, E. Lauga, Phys. Rev. Lett. 101, 038102 (2008)

41. D. Takagi, J. Palacci, A.B. Braunschweig, M.J. Shelley, J. Zhang, Soft Matter 10, 1784 (2014)

42. I.O. Götze G. Gompper, Phys. Rev. E 82, 041921 (2010)

43. T. Ishikawa, M.P. Simmonds, T.J. Pedley, J. Fluid Mech. 568, 119 (2006)

44. K. Ishimoto, E.A. Gaffney, Phys. Rev. E 88, 062702 (2013)

45. G.-J. Li, A.M. Ardekani, Phys. Rev. E 90, 013010 (2014)

46. J. Elgeti, U.B. Kaupp, G. Gompper, Biophys. J. 99, 1018 (2010) 
47. B.M. Friedrich, F. Jülicher, New J. Phys. 10, 123025 (2008)

48. B.M. Friedrich, F. Jülicher, Phys. Rev. Lett. 103, 068102 (2009)

49. I.H. Riedel, K. Kruse, J. Howard, Science 309, 300 (2005)

50. Y. Yang, F. Qiu, G. Gompper, Phys. Rev. E 89, 012720 (2014)

51. K. Miki D.E. Clapham, Curr. Biol. 18, 443 (2013)

52. V. Kantsler, J. Dunkel, M. Blayney, R.E. Goldstein, eLife 3, e02403 (2014)

53. K. Ishimoto, E.A. Gaffney, J. Roy. Soc. Interface 12, 20150172 (2015)

54. H. Löwen, Eur. Phys. J. Special Topics 225, 3019 (2016)

55. H. Stark, Eur. Phys. J. Special Topics 225, 3069 (2016)

56. L. Lemelle, J.-F. Palierne,E. Chatre, C. Vaillant, C. Place, Soft Matter 9, 9759 (2013)

57. L. Lemelle, J. Palierne, E. Chatre, C. Place, J. Bacteriol. 192, 6307 (2010)

58. R. Di Leonardo, D. Dell'Arciprete, L. Angelani, V. Iebba, Phys. Rev. Lett. 106, 038101 (2011)

59. K.M. Ottemann, J.F. Miller, Mol. Microbiol. 24, 1109 (1997)

60. L.A. Pratt, R.R. Kolter, Mol. Microbiol. 30, 285 (1998)

61. Podcast "Welt der Physik, Folge 189 - Mikroschwimmer im Modell" (09. Juli 2015); http://www.weltderphysik.de/mediathek/podcast/.

62. I.O. Götze, G. Gompper, EPL 92, 64003 (2010)

63. J. Hu, A. Wysocki, R.G. Winkler, G. Gompper, Sci. Rep. 5, 9586 (2015)

64. J. Hu, M. Yang, G. Gompper, R.G. Winkler, Soft Matter 11, 7867 (2015)

65. G.-J. Li, A. Karimi, A.M. Ardekani, Rheol. Acta 53, 911 (2014)

66. X. Yang, M.L. Manning, M.C. Marchetti, Soft Matter 10, 6477 (2014)

67. S.C. Takatori, W. Yan, J.F. Brady, Phys. Rev.Lett. 113, 028103 (2014)

68. A.P. Solon, J. Stenhammar, R. Wittkowski, M. Kardar, Y. Kafri, M.E. Cates, J. Tailleur, Phys. Rev. Lett. 114, 198301 (2015)

69. A.P. Solon, Y. Fily, A. Baskaran, M.E. Cates, Y. Kafri, M. Kardar, J. Tailleur, Nat. Phys. 11, 673 (2015)

70. R.G. Winkler, A. Wysocki, G. Gompper, Soft Matter 11, 6680 (2015)

Open Access This is an Open Access article distributed under the terms of the Creative Commons Attribution License (http://creativecommons.org/licenses/by/4.0), which permits unrestricted use, distribution, and reproduction in any medium, provided the original work is properly cited. 